\title{
Planos Plurianuais Estaduais (2016-2019). Uma proposta de avaliação de sua adequação ao planejamento estratégico
}

\section{Juan Vicente Bachiller}

Universidade Federal Fluminense (UFF), Rio de Janeiro, RJ - Brasil

O presente artigo busca elaborar um diagnóstico da implantação do planejamento estratégico nos governos estaduais. Para isso, centra-se na análise dos Planos Plurianuais (PPAs) dos estados, durante o período de 2016-2019, elaborando um índice para medir o grau em que esses planos atingem as pautas necessárias para auxiliar o ciclo de gestão. O objetivo é analisar a adequação dos PPAs estaduais aos atributos do planejamento estratégico, descrevendo em que medida são um simples documento formal para cumprir o procedimento legal, ou se, de fato, trata-se de ferramentas que possam auxiliar em uma gestão pública focada na provisão eficaz e eficiente de bens e serviços públicos. Para isso, são avaliadas as dimensões estratégica, operativa, programática e de integração orçamentária, estabelecendo indicadores para comparar o grau de evolução dessa técnica de administração. Os resultados mostram um insuficiente desenvolvimento do planejamento estratégico, sendo que os PPAs, em sua maioria, têm sido concebidos como orçamentos para um período de quatro anos, e não como uma ferramenta de gestão.

Palavras-chave: planejamento estratégico, governos subnacionais, federalismo 


\section{Planes Plurianuales Estatales (2016-2019). Una propuesta de evaluación de su adecuación a la planificación estratégica}

El presente artículo pretende elaborar un diagnóstico de la implantación de la planificación estratégica en los gobiernos estatales. Para eso, se centra en el análisis de los Planos Plurianuales (PPAs) de los estados, durante el período de 2016-2019, elaborando un índice para medir el grado en que éstos alcanzan las pautas necesarias para auxiliar en el ciclo de gestión. El objetivo es analizar la adecuación de los PPAs estatales a los atributos de la planificación estratégica, describiendo en qué medida los PPAs estatales son un simple documento formal para cumplir con el procedimiento legal, o si se tratan de herramientas que puedan auxiliar una gestión pública enfocada en la provisión eficaz y eficiente de bienes y servicios públicos. Para eso, serán evaluadas las dimensiones estratégica, operativa, programática y de integración presupuestaria, estableciendo indicadores para comparar el grado de evolución de esta técnica de administración. Los resultados muestran un insuficiente desarrollo de la planificación estratégica, dado que la mayoría de los PPAs están concebidos como presupuestos para un periodo de cuatro años, y no como una herramienta de gestión.

Palabras clave: planificación estratégica, gobiernos subnacionales, federalismo

\section{State Pluriannual Plans (2016-2019). A proposal of evaluation about its accuracy to strategic planning}

This article aims to develop a diagnosis of the implementation of strategic planning in the state governments. For that, it focuses on the analysis of the states' Pluriannual Plans (PAPs) during the 2016-2019 period, preparing an index to measure the degree to which they reach the necessary guidelines to assist in the management cycle. The objective is to analyze states Pluriannual Plans adequation to strategic planning attributes, by describing to what extent the state PPAs are a simple formal document to comply with the legal procedure, or if they are tools that can assist a public management focused on the effective and efficient provision of public goods and services. For that, the strategic, operative, programmatic and budgetary integration dimensions will be evaluated, establishing indicators to compare the degree of evolution of this administration technique. The results show an insufficient development of strategic planning, since most of the PPAs are conceived as budgets for a period of four years, and not as a management tool.

Keywords: strategic planning, subnational governments, federalism 


\section{Introdução}

O planejamento estratégico é uma ferramenta associada ao paradigma da administração pública pós-garantista que, para além de cumprir a legalidade vigente e proteger o cidadão da arbitrariedade do Estado, procura fornecer bens e serviços públicos essenciais de acordo com os princípios de efetividade e eficiência, com padrões de qualidade que sejam efetivamente medíveis. Nascido no âmbito da administração privada, o planejamento estratégico tem como alvo substituir o foco no cumprimento dos processos e procedimentos legais pela prioridade por atingir metas e objetivos consistentes, com a missão e visão de longo prazo estabelecidas pela organização. Aplicado ao setor público, é um dos cinco subsistemas que, segundo o Banco Interamericano de Desenvolvimento, integram o ciclo da gestão por resultados (GpR), formado por planejamento orientado a resultados, orçamento por resultados, gestão financeira pública, gestão de programas e projetos e, por último, monitoramento e avaliação (KAUFMANN; SANGINÉS; GARCíA MORENO, 2015).

Desses cinco subsistemas, o primeiro deles - o planejamento - tem o papel de orientar estrategicamente e de forma prospectiva as futuras ações no ciclo da GpR. Por ser a primeira das etapas do sistema, o desempenho das seguintes ficará condicionado pela correta elaboração tanto de um planejamento estratégico de longo prazo, como de um planejamento operacional de limite temporal mais curto.

A história do planejamento governamental no Brasil antecede a implantação das técnicas de gestão pública, tendo a sua origem nos planos de desenvolvimento econômico da década de 1940 e 1950 (ALMEIDA, 2006), evoluindo posteriormente como ferramenta para subsidiar a elaboração orçamentária e, como tal, é consagrado no texto constitucional de 1988 (PARES; VALLE, 2006). Considera-se que, em sentido estrito, o planejamento como elemento da gestão pública somente começou a ser implantado a partir do Plano Plurianual (PPA) do Governo Federal para o período de 2000 a 2003. Nesse plano, integram-se, pela primeira vez, os instrumentos de planejamento, orçamento e gestão, organizando-se suas ações numa perspectiva estratégica de quatro anos, de forma que elas possam ser executadas na Lei Orçamentária Anual como programas, posteriormente acompanhados e avaliados por meio de indicadores (GARCÉS; SILVEIRA, 2002). Desde então, o planejamento 
plurianual, como instrumento ao serviço de uma gestão eficaz e eficiente feito pela União, é a etapa mais desenvolvida pelos governos dos países latino-americanos, revelando-se com alto desempenho em sua capacidade estratégica e operativa (GARCíA LóPEZ; GARCíA MORENO, 2010).

Tomando-se o desenvolvimento do planejamento plurianual como ferramenta para auxiliar a gestão pública no nível nacional, cabe se perguntar se essa boa prática da administração pública terá se espalhado da mesma forma em outros níveis de governo. Ou seja, dada a obrigatoriedade de implementar um PPA para todas as unidades federativas, segundo a Constituição de 1998, questiona-se se os estados da Federação têm acompanhado a evolução do planejamento como ferramenta do ciclo de gestão, ou têm se limitado a apresentar um orçamento genérico para um período quatrienal.

O objetivo deste artigo, em suma, é analisar o desenvolvimento do planejamento estratégico nos estados brasileiros, empregando para isso uma avaliação de como este é aplicado nos seus PPAs. A intenção é conhecer quais estados estão mais perto de fornecer uma ferramenta para auxiliar o ciclo da gestão pública, e quais se limitam à prática de construir um PPA como simples ritual procedimental. Para isso, será criado um índice que mensure a inserção de cada PPA dentro das premissas do planejamento estratégico de acordo com quatro dimensões: estratégica, programática, operacional e, por último, de integração com o orçamento. Trata-se de uma abordagem exploratória descritiva, que busca responder à pergunta de quais são os estados que mais têm desenvolvido técnicas de gestão estratégica na elaboração dos seus PPAs.

Juntamente com esta introdução e um apartado final de conclusões, este artigo se estrutura em três apartados. No primeiro deles, de caráter teórico, será revisada a literatura sobre o planejamento estratégico e a sua aplicação nos distintos níveis federativos no Brasil, juntamente com um estado da arte dos estudos sobre os PPAs, nos níveis federal e estadual. No segundo apartado, de caráter metodológico, será desenvolvida a operacionalização de cada uma das dimensões e variáveis que compõem o índice, embasando teoricamente a justificativa da escolha de cada uma delas. No terceiro e último apartado, serão apresentados os resultados da pesquisa. 


\section{Planejamento Estratégico e Planejamento Plurianual na Administração Pública}

Os escritos de Peter Drucker $(1954,1964)$ constituem o início das reflexões sobre a gestão por objetivos na administração de empresas. Suas obras The practice of management e Managing for results representaram uma revolução na gerência privada, popularizando mundialmente os conceitos de direção por objetivos e estratégia de negócios. Desde então, considera-se o planejamento estratégico aplicado à gestão por objetivos como um processo de definição de grandes metas a serem alcançadas pela organização, as quais serão descompostas numa sequência lógica de ações e programas subordinados a esses objetivos superiores, monitorados e avaliados por um conjunto de indicadores que medem o grau em que são atingidos em sequências temporais preestabelecidas.

A gestão por objetivos, no setor público, começa a ser implantada na década de 1980, em países como Austrália, Inglaterra e Nova Zelândia, relacionada ao conjunto de reformas para inserir o posteriormente conhecido paradigma da Nova Gestão Pública (NGP) (TALBOT, 2007). Na Inglaterra, segundo Pollitt (2018), a sua origem está na implantação dos primeiros sistemas de indicadores para medir o desempenho no National Health Service (NHS); evoluindo posteriormente, durante o período dos governos trabalhistas - 19972010 - à sua massiva implantação em toda a administração pública. Nos Estados Unidos, tais reformas aconteceram no contexto da implantação da NGP durante o Governo Clinton, quando foi aprovada, em 1993, a Government Performance and Results Act 1993 -, introduzindo, nas agências federais, a obrigatoriedade da gestão baseada no estabelecimento de objetivos e a mensuração de resultados (NATHAN, 2001).

Dessa forma, a gestão por objetivos foi introduzida nas rotinas da administração pública, de maneira gradual, mas desigualmente nos distintos países e nos diferentes níveis de governo. Pode ser caracterizada como um método para usar as técnicas de planejamento, orçamento, gerência e avaliação, identificando as necessidades governamentais, desenvolvendo um plano e um conjunto de políticas relacionadas, e criando um conjunto de indicadores para acompanhar o processo. Segundo Pollit e Bockhaert (2001), suas principais características mantêm o foco no desempenho, em sua medição, nos incentivos para atingir os objetivos prefixados e na aplicação de técnicas 
gerenciais. Embora possa variar segundo o autor ou segundo a prática empregada, a gestão por objetivos no setor público se compõe, basicamente, das seguintes etapas: planejamento estratégico, orçamento por resultados, processos de gerenciamento, avaliação de resultados e reporte dos resultados (WALTERS; ABRAHAMS; FoUNTAIN, 2003). Neste ponto, é importante destacar que, embora a implementação da gestão por objetivos tenha sido influenciada inicialmente pelas técnicas gerencialistas do NPM, com o passar do tempo, adaptou-se às rotinas da administração neoweberiana, sendo hoje reconhecida como importante capacidade burocrática dos modernos Estados (DUSSAGE, 2016).

Na América Latina, a expansão da gestão por objetivos tem um forte vínculo com uma orientação a favor do planejamento estratégico e, como tal, tem sido influenciada por diferentes organismos internacionais, como a Organização das Nações Unidas (ONU), a Organização para a Cooperação e o Desenvolvimento Econômico (OCDE), o Banco Mundial (BM), o Fundo Monetário Internacional (FMI) e o Banco Interamericano de Desenvolvimento (BID) (CHICA, 2015). É esta última instituição a que mais tem se comprometido com o desenvolvimento do planejamento estratégico na região, por meio de sua divisão de capacidade institucional, criando o sistema Prodev, que acompanha sua implantação nos diferentes países (KAUFMAN; MORENO; SANGINÉS, 2015). Outro dos organismos responsáveis pelo assessoramento e acompanhamento da implantação do planejamento estratégico é o Centro Latinoamericano de Administración para el Desarrollo (Clad), o qual realiza avaliações periódicas sobre o seu desenvolvimento nos países da região (CLAD, 2007; CUNILL; OSPINA, 2012). Evidencia-se, assim, um processo de aprendizado internacional que ajudou a difundir as reformas do planejamento estratégico associado à gestão por objetivos na América Latina, principalmente nos países que primeiramente adotaram essa política, como o Brasil, o Chile e o México (DuSSAUGE, 2015; MILANESI, 2018).

No Brasil, o planejamento estratégico, como ferramenta de gestão pública, começa a ser adotado por ocasião das reformas administrativas impulsionadas pelo ministro Bresser-Pereira, desde o Ministério da Administração Federal e a Reforma do Estado (Mare). Na onda de uma maior desconcentração, foram implementados novos mecanismos de contratualização por resultados e avaliação das políticas públicas (ABrucio; SEgatTo, 2017). Como foi mencionado, o planejamento estratégico associado à gestão por objetivos foi introduzido por ocasião do PPA 2000-2003 “Avança Brasil”, que, 
em função da integração com a Lei de Responsabilidade Fiscal e com um planejamento de mais longo prazo recolhido no documento "Eixos Nacionais de Integração e Desenvolvimento", organizou as ações de investimento público em 365 programas que serviriam como base à elaboração dos sucessivos orçamentos anuais. Nessa nova concepção, o planejamento deixa de ser a extrapolação plurianual do orçamento, para se transformar, mediante a elaboração de programas, para atingir objetivos específicos, subordinados a uma meta geral, em uma ferramenta com o objetivo de solucionar problemas públicos concretos, integrando plano, ações orçamentárias e medição do desempenho (GARCÉS; SILVEIRA, 2002).

A metodologia da gestão por objetivos estabelece que o planejamento estratégico é a primeira das etapas do ciclo de gestão, de forma que se converta no elemento estruturante de todo o processo. É importante levar em conta que o planejamento governamental tem sua gênese anterior à implantação da gestão por objetivos no setor público. Os primeiros planos de governo têm origem nos planos quinquenais da União Soviética, relacionados ao paradigma do dirigismo econômico estatal. Posteriormente, o planejamento governamental se estende às economias capitalistas, inclusive à dos Estados Unidos, durante a época do New Deal, sendo característicos os grandes planos de desenvolvimento em países como a França e Japão. A lógica subjacente é que os investimentos necessários, para acompanhar o processo de mudança estrutural inerente à industrialização tardia, precisam ser estabelecidos ou, no mínimo, indicados pelo Estado. Posteriormente, a crise do modelo dirigista, unida à generalização do modelo de planejamento estratégico dentro das empresas, a partir da década de 1970, trouxe uma mudança na orientação do planejamento governamental, tornando-o uma ferramenta para subsidiar no controle das contas públicas. Somente então, a partir das mencionadas reformas do NPM, o planejamento governamental passa a ser uma ferramenta de gestão pública, com a função de ajudar na elaboração e acompanhamento de políticas públicas para que objetivos relevantes de transformação social fossem atingidos.

A história do planejamento governamental no Brasil corresponde, de modo geral, a essa sucessão de etapas. Em um primeiro momento, o planejamento é encaminhado por meio da direção estatal do processo de desenvolvimento econômico e de industrialização do país, sendo característicos, entre outros, o Programa de Metas e os sucessivos Planos Nacionais de Desenvolvimento Econômico da década de 1970. Ao mesmo tempo, a 
capacidade estatal para acompanhar o processo se estendeu com a instauração de organismos como o Dasp, o Ipea, a Secretaria de Estado de Planejamento, ou o denominado Sistema Federal de Planejamento (DE TONI, 2016). Em um segundo momento, já durante a época da redemocratização e da crise da década de 1980, acontece um desmantelamento desse sistema, que ficou subordinado à lógica do controle fiscal e inflacionário, ditada pelo Ministério da Fazenda (REzende, 2011). Nesse período, entretanto, a Constituição de 1988 sinaliza a obrigatoriedade do planejamento em todos os entes federativos e a sua integração por meio do sistema constituído pelo Plano Plurianual, a Lei de Diretrizes Orçamentárias (LDO) e a Lei Orçamentária Anual (LOA). Entretanto, somente no plano 2000-2003 é que se imprime ao orçamento uma perspectiva mais gerencial, com o estabelecimento de objetivos estratégicos, identificação de problemas e oportunidades, concepção de programas, especificação de ações, atribuição de indicadores, e avaliação de resultados, entre outros (GARCIA, 2015a). Desde então, os sucessivos PPAs do Governo Federal têm melhorado sua técnica, adquirindo uma verdadeira função de orientação estratégica (COUTO, 2017), embora ainda persista uma tendência a misturá-lo com a prospecção quatrienal da LOA, o que faz diluir a sua capacidade como ferramenta de gestão (DE TONI, 2017).

No nível subnacional, esse lento e incompleto processo da instauração do planejamento estratégico como ferramenta de gestão pública tem o seu reflexo nos PPAs dos estados. Segundo Abrucio e Gaetani (2008), embora tenha se desenvolvido um movimento em favor do seu fortalecimento, impulsionado pelos resultados do PPA federal de 2000-2003, pouco tinha sido conseguido na articulação de políticas mediante a gestão por programas. Por sua vez, o estudo do Ipea Planejamento e Gestão Governamental na Esfera Estadual trouxe uma série de diagnósticos sobre os PPAs, do período 2011-2015, com um total de dez estados (Bahia, Ceará, Espírito Santo, Minas Gerais, Mato Grosso do Sul, Paraná, Rio de Janeiro, Rio Grande do Norte, Rio Grande do Sul e São Paulo); com resultados desiguais entre eles. Entre as conclusões gerais a serem extraídas desse estudo, cabe mencionar, segundo Carneiro (2015), que a maioria dos PPAs analisados responde a uma lógica de se fazer cumprir a formalidade de elaborar a programação orçamentária, com escasso interesse estratégico, concedido por parte dos governos, em seu uso na gestão. 
Tendo em conta o que foi analisado até aqui, cabe questionar, por um lado, se o processo de expansão do planejamento estratégico, por meio dos PPAs, nos distintos estados brasileiros, tem evoluído durante o período 2016-2019, ou se persistem as principais falhas apontadas acima. De outro lado, cabe estabelecer uma comparação mais abrangente, que tome a todos os estados brasileiros como amostra, para se entender, com maior precisão, como está acontecendo esse fenômeno. Tais questionamentos serão respondidos nos próximos apartados, por intermédio da elaboração do índice de penetração da GpR nos PPAs estaduais no mencionado período.

\section{Como medir a implantação do planejamento estratégico nos PPAs estaduais?}

Como exposto até este ponto, o PPA é explicitado na Carta Magna de 1988 com a intenção, por parte dos constituintes, de que os diferentes governos (federal, estaduais e municipais) sistematizassem os seus programas de investimento, com horizontes temporais definidos, desde o segundo ano do mandato até o primeiro ano do mandato sucessivo. Desse modo, pretendia-se organizar, por um período de quatro anos, as despesas que posteriormente seriam anualmente contempladas na LDO e na LOA, tendo como resultado um orçamento plurianual dos investimentos do setor público. A partir de 1998, no contexto das reformas gerenciais do Estado, a técnica de planejamento passa a ser compreendida não simplesmente com um objetivo fiscal de controle plurianual das contas públicas, mas de acordo com as suas potencialidades, no sentido de definir estrategicamente as linhas do programa do governo da União. Para isso, estabeleceu-se que o PPA tivesse como unidade básica de organização e de integração com a LDO e a LOA o programa formado por ações, com objetivos e diretrizes a serem atingidos, metas e custos quantificados, e avaliação do desempenho. O caminho para estabelecer o PPA, como a primeira das etapas do ciclo de gestão, no setor público, entretanto, não tem estado isento de limitações, com uma forte tendência, no nível da União, a continuar usando-o como uma programação plurianual de despesas (GARCIA, 2015b).

Assim, não é difícil pensar que, no nível subnacional, a elaboração do planejamento estratégico possa ter um desenvolvimento incompleto e, muito provavelmente, desigual entre as distintas unidades federativas. Para responder às perguntas de qual é esse grau 
de desenvolvimento no nível estadual e quais são os estados que mais têm desenvolvido o seu PPA como ferramenta de acordo com o paradigma do planejamento estratégico, em primeiro lugar, devemos responder à pergunta de qual é o procedimento para se estabelecer essa técnica dentro do setor público. Após respondê-la, serão reunidas as condições para se chegar a um índice com as principais dimensões e variáveis que possam medir essa questão.

Bryson (2004) estabelece que o planejamento estratégico governamental precisa contar com um mandato e uma missão da organização, uma visão organizacional do futuro, uma análise ambiental das fraquezas, fortalezas, ameaças e oportunidades, e um conjunto limitado de objetivos estratégicos que, mediante a técnica do balanced scorecard, estabeleça planos de ação para atingir os objetivos. Kaufman, Sanginés e Garcia Moreno (2015) fazem uma operacionalização da qualidade do planejamento governamental de acordo com três dimensões: 1) capacidade estratégica do planejamento (existência de planos nacionais de médio prazo e setoriais, e indicadores para acompanhar o cumprimento de metas); 2) operatividade do planejamento (identificação de programas, produtos, unidades responsáveis e a sua integração com os programas do orçamento anual); e, por último, 3) participação societal no processo. Por sua vez, o Modelo abierto de Gestión para Resultados en el sector público, desenvolvido pelo Clad (2011), estabelece como principais componentes do ciclo de planejamento a existência de um sistema de informação para formular e monitorar o plano, o estabelecimento de programas e de metas, e os objetivos de médio prazo, que estes objetivos possam ser medidos, e que existam unidades responsáveis por cada um deles. Por último, Garcia (2015b), citando as pautas estabelecidas pelo Manual Técnico do Orçamento, após a reforma de 1998, indica, como elementos essenciais do plano, os objetivos estratégicos, os problemas e as oportunidades, os programas, as ações dos programas com identificação dos produtos, os indicadores atribuídos aos objetivos, os gerentes dos programas, a avaliação e a prestação de contas.

Com base nessas quatro definições, desenvolveu-se neste artigo uma operacionalização da qualidade dos PPAs de acordo com os pressupostos do planejamento estratégico, estabelecendo-se quatro dimensões. A primeira delas é a dimensão estratégica, que corresponde à ideia de que o plano de governo deve estabelecer um diagnóstico geral, no qual se encontra a organização - o governo 
estadual, neste caso - e para onde se pretende ir - na transformação social do estado -, de acordo com as fraquezas e as fortalezas internas da organização, as ameaças e as oportunidades externas do ambiente social, político e econômico. A segunda, é a dimensão estruturante, a qual corresponde à ideia de que a primeira dimensão deve ser executada, estabelecendo-se um conjunto de objetivos prioritários que, por sua própria definição, deve discriminar as grandes linhas do programa, de acordo com aqueles grandes conjuntos de políticas que são tidos como essenciais para tornar executiva a estratégia. A terceira delas é a dimensão programática e tem a ver com a precisão com que os objetivos prioritários estão desagregados em um conjunto de programas, na qual sejam estabelecidos objetivos concretos de políticas públicas, com estabelecimento de recursos, projetos e ações, e indicadores para medir o grau de consecução da meta. A quarta e última das dimensões é a operativa e tem a ver com a forma com que o PPA estabelece ferramentas para se integrar com a LDO e a LOA, servindo assim como transição entre os objetivos estratégicos e uma programação anual operativa.

Cada uma das quatro dimensões tem um peso similar num índice que vai de 0 a 1 , ou seja, a cada uma delas corresponde um coeficiente de 0,25. Por sua vez, cada um dos indicadores terá um peso similar dentro da sua dimensão. Os indicadores para medir a dimensão estratégica são três: existência ou não de uma missão; existência ou não de uma visão; e análise FOFA ( $F$ - forças; O - oportunidades; F - fraquezas; A - ameaças) para estabelecer tal estratégia. Com esses indicadores, pretende-se avaliar o grau em que cada PPA tem sido capaz de captar o mandato e os propósitos fundamentais do governo do estado, a ideia de transformação social que ele projeta para o futuro e, por último, a forma com que essa transformação pode ser atingida de acordo com a previsão do entorno externo institucional, junto com as capacidades administrativas do governo.

Os indicadores para medir a dimensão estruturante são três: 1) a existência ou não de um número razoável, entre 3 e 5 , de objetivos; 2 ) que os objetivos derivem da estratégia refletida na missão e visão; e 3) que estejam priorizados ou hierarquizados. A ideia geral da dimensão é conhecer em que medida o PPA é capaz de tornar passível de execução a missão e a visão por meio da priorização das linhas principais da ação do governo.

Os dois primeiros indicadores pretendem avaliar o grau em que o PPA estabelece as grandes linhas estratégicas do governo e como elas dependem da dimensão anterior. 
O último dos indicadores se refere à forma com que tais objetivos, por sua vez, fornecem uma escala de prioridades entre eles e se são desagregados em uma série de objetivos (secundários, de menor importância hierárquica). Assim, este último indicador pretende avaliar como a dimensão se articula com a seguinte, que é a estruturação em programas do PPA, dado que cada um deles deveria derivar de algum dos objetivos estruturadores.

Os indicadores para medir a dimensão programática são cinco: 1) definição ou não de objetivos para os programas; 2 ) existência de unidade ou unidades administrativas responsáveis por cada programa; 3) uso de indicadores de acompanhamento para as metas do programa; 4) desagregação anual desses indicadores; e, por último, 5) desagregação ou não desses programas em projetos e atividades diferenciadas. Os indicadores dessa dimensão revelam o quanto o PPA cumpre com as caraterísticas fundamentais da técnica do orçamento por programas orientado a resultados. Tais características congregam um objetivo qualitativo, uma meta quantitativa e um gestor responsável por eles, o que se reflete nos três primeiros indicadores. O quarto indicador contempla o grau de detalhamento em que a meta é acompanhada, em cada exercício orçamentário anual. O último indicador apreende o grau em que cada programa se subdivide, em projetos ou em atividades.

Por último, a dimensão operativa é constituída de três indicadores: a alocação, no PPA, de um financiamento para cada programa; que esse financiamento esteja desagregado anualmente; e, por último, que os programas do PPA tenham o seu programa correlato na LOA de cada ano. Esses indicadores apontam o grau em que o PPA pode se transformar num plano operativo para cada um dos anos em que permanecer vigente. Os dois primeiros avaliam o grau de detalhe em que cada programa tem alocada uma estimativa de despesa. O terceiro indicador analisa se o código destinado a cada programa no PPA corresponde a um código na LOA, e se, portanto, tem possibilidades reais para ser executável. 


\section{Quadro 1 | Operacionalização}

Dimensão estratégica (D1). (peso 0,25 pontos)
var1. O PPA define uma missão? (sim: 0,08 pontos/ não: 0 pontos)
var2. O PPA define uma visão de futuro? (sim: 0,08 pontos/ não: 0 pontos)
var3. A estratégia está elaborada a partir de uma análise FOFA?
(sim: 0,08 pontos/não: 0 pontos)
Dimensão estruturante (D2). (peso 0,25 pontos)
var4. Os objetivos estratégicos derivam da dimensão estratégica?
(sim: 0,08 pontos/ não: 0 pontos)
var5. Estão priorizados e/ou hierarquizados? (sim: 0,08 pontos/ não: 0 pontos)
var6. Existe um número consequente de entre 3 a 5 objetivos? (sim: 0,08 pontos/ não: 0
pontos)
Dimensão programática (D3). (peso 0,25 pontos)
var7. Os programas têm objetivos definidos? (sim: 0,05 pontos/não: 0 pontos)
var8. Os programas indicam a unidade responsável? (sim: 0,05 pontos/não: 0 pontos)
var10. Os programas têm indicadores de resultado? (sim: 0,05 pontos/não: 0 pontos)
var11. Os programas são desagregados em projetos e atividades diferenciadas? (sim: 0,05
pontos/não: 0 pontos)
var14. Os programas do PPA têm o seu correlato no orçamento programa? (sim: 0,08 pontos/
não: 0 pontos)
var12. Os programas têm financiamento assignado? (sim: 0,08 pontos/ não: 0 pontos)
Dimensão operativa (D4). (peso 0,25 pontos)

Fonte: elaboração própria.

\section{Diagnóstico dos PPAs estaduais}

Uma vez realizada a operacionalização, estabelecendo as dimensões e variáveis que compõem o índice de qualidade dos PPAs estaduais, é possível medir esse fenômeno e proceder às comparações. Espera-se, de um lado, estabelecer quais estados apresentam um melhor e um pior desempenho em seu planejamento estratégico e, de outro lado, quais são os aspectos em que o planejamento mostra as suas fortalezas e fraquezas. 
A primeira dimensão, a estratégica, é a que mostra o pior desempenho dentre as quatro aqui analisadas, atingindo uma média de somente 0,06 pontos em um total de 0,25, ou seja, menos de um terço do máximo objetivo possível. Destacam-se negativamente as variáveis da missão e da análise FOFA, sendo que a definição de uma visão também não é uma prática plenamente generalizada. Apenas Amapá e Sergipe contam com uma missão definida, e somente Mato Grosso utiliza a metodologia FOFA para ajudar a definir a sua estratégia, incluindo o PPA do Rio Grande do Norte, neste indicador, por usar a metodologia da análise prospectiva de cenários. Embora a maioria dos PPAs afirme fazer uma análise de cenário, na realidade, eles se limitam a compilar uma série de dados sobre conjuntura econômica e social, internacional, nacional e estadual, mas sem muita conexão com a natureza prospetiva do plano. Por sua vez, a variável visão de futuro é a que melhor se encontra nessa dimensão, com quinze estados que incorporam esse elemento para definir o seu planejamento estratégico.

Cabe considerar que alguns estados têm desenvolvido planos estratégicos de mais longo prazo do que o PPA, como no caso de Mato Grosso, Paraíba, Rondônia e Santa Catarina. Esses estados têm realizado, seja a sua análise FOFA, seja a definição da sua visão em documentos, em lugar de fazê-lo no PPA, mas, ainda assim, também foram pontuados de forma positiva. Por último, é importante mencionar que nenhum estado atingiu a pontuação máxima na dimensão estratégica, enquanto 11 estados não atingiram nenhum ponto nela, sendo que apenas quatro PPAs - Mato Grosso, Pernambuco, Rio Grande do Norte e Sergipe - tiveram ao menos duas variáveis bem avaliadas. 
Quadro 2 | D1. Dimensão estratégica (0,25 pontos)

\begin{tabular}{|c|c|c|c|c|}
\hline Estado & var1 & var2 & var3 & Pontos \\
\hline Acre & Não & Sim & Não & 0,08 \\
\hline Alagoas & Não & Sim & Não & 0,08 \\
\hline Amapá & Sim & Não & Não & 0,08 \\
\hline Amazonas & Não & Não & Não & 0 \\
\hline Bahia & Não & Não & Não & 0 \\
\hline Ceará & Não & Não & Não & 0 \\
\hline Distrito Federal & Não & Sim & Não & 0,08 \\
\hline Espírito Santo & Não & Sim & Não & 0,08 \\
\hline Goiás & Não & Não & Não & 0 \\
\hline Maranhão & Não & Não & Não & 0 \\
\hline Mato Grosso & Não & Sim & $\operatorname{Sim}\left({ }^{*}\right)$ & 0,17 \\
\hline M. Grosso do S. & Não & Sim & Não & 0,08 \\
\hline Minas Gerais & Não & Não & Não & 0 \\
\hline Pará & Não & Sim & Não & 0,08 \\
\hline Paraíba & Não & $\operatorname{Sim}(*)$ & Não & 0,08 \\
\hline Paraná & Não & Não & Não & 0 \\
\hline Pernambuco & Não & Sim & Sim & 0,17 \\
\hline Piauí & Não & Sim & Não & 0,08 \\
\hline Rio de Janeiro & Não & Não & Não & 0 \\
\hline R.G. do Norte & Não & Sim & $\operatorname{Sim}(* *)$ & 0,17 \\
\hline R.G. do Sul & Não & Sim & Não & 0,08 \\
\hline Rondônia & Não & $\operatorname{Sim}(*)$ & Não & 0,08 \\
\hline Roraima & Não & Não & Não & 0 \\
\hline Santa Catarina & Não & $\operatorname{Sim}(*)$ & Não & 0,08 \\
\hline São Paulo & Não & Não & Não & 0 \\
\hline Sergipe & Sim & Sim & Não & 0,17 \\
\hline Tocantins & Não & Não & Não & 0 \\
\hline
\end{tabular}

Fonte: elaboração própria.

A dimensão estruturante melhora em desempenho em relação à primeira dimensão, embora a pontuação média atingida de 0,12 fique abaixo da metade da maior pontuação possível. Oito estados - Alagoas, Distrito Federal, Mato Grosso, Mato Grosso 
do Sul, Pará, Rio Grande do Norte, Rio Grande do Sul e Sergipe - atingiram o maior desempenho possível, e isso significa que conseguiram estabelecer em seus PPAs uma priorização de quais linhas de políticas públicas seriam decisivas para a sua estratégia. Porém, cabe destacar negativamente que oito estados - Ceará, Minas Gerais, Paraíba, Paraná, Rondônia, Roraima, Santa Catarina e Tocantins, não pontuaram em nenhuma das variáveis, considerando-se, assim, que foram incapazes de priorizar um conjunto de políticas sobre as demais para atingir seus objetivos estratégicos. Levando-se em conta as variáveis, cabe mencionar que somente 11 estados relacionam os seus objetivos estratégicos com a visão de futuro, contemplada na dimensão estratégica, realizando de forma correta a metodologia do planejamento orientado a resultados. Por sua vez, um pouco mais da metade dos PPAs, em torno de 15 e 14 respectivamente, desenvolvem, de um lado, objetivos estratégicos que se desagregam depois em objetivos secundários, dando lugar aos programas na dimensão seguinte e, de outro lado, conseguem priorizar esses objetivos em ordem de relevância.

\section{Quadro 3 | D2. Dimensão estruturante $(0,25)$}

\begin{tabular}{|l|c|c|c|c|}
\hline Estado & var4 & var5 & var6 & Pontos \\
\hline Acre & Sim & Não & Não & 0,08 \\
\hline Alagoas & Sim & Sim & Sim & 0,25 \\
\hline Amapá & Não & Não & Sim & 0,08 \\
\hline Amazonas & Não & Sim & Sim & 0,17 \\
\hline Bahia & Não & Sim & Não & 0,08 \\
\hline Ceará & Não & Não & Não & 0 \\
\hline Distrito Federal & Sim & Sim & Sim & 0,25 \\
\hline Espírito Santo & Sim & Sim & Não & 0,17 \\
\hline Goiás & Não & Sim & Sim & 0,17 \\
\hline Maranhão & Não & Sim & Não & 0,08 \\
\hline Mato Grosso & Sim & Sim & Sim & 0,25 \\
\hline M. Grosso do S. & Sim & Sim & Sim & 0,25 \\
\hline Minas Gerais & Não & Não & Não & 0 \\
\hline Pará & Sim & Sim & Sim & 0,25 \\
\hline Paraíba & Não & Não & Não & 0 \\
\hline Paraná & Não & Não & Não & 0 \\
\hline Pernambuco & Não & Sim & Sim & 0,17 \\
\hline
\end{tabular}




\begin{tabular}{|l|c|c|c|c|}
\hline Piauí & Sim & Não & Sim & 0,17 \\
\hline Rio de Janeiro & Não & Não & Sim & 0,08 \\
\hline R.G. do Norte & Sim & Sim & Sim & 0,25 \\
\hline R.G. do Sul & Sim & Sim & Sim & 0,25 \\
\hline Rondônia & Não & Não & Não & 0 \\
\hline Roraima & Não & Não & Não & 0 \\
\hline Santa Catarina & Não & Não & Não & 0 \\
\hline São Paulo & Não & Sim & Não & 0,08 \\
\hline Sergipe & Sim & Sim & Sim & 0,25 \\
\hline Tocantins & Não & Não & Não & 0 \\
\hline & & & & $\mu=0,12$ \\
\hline
\end{tabular}

Fonte: elaboração própria.

A dimensão programática atinge uma pontuação de 0,14 sobre 0,25 , acima da metade do indicador, mostrando que, na medida em que as dimensões se afastam da formulação estratégica, aproximando-se da execução orçamentária, o desempenho aumenta. O Distrito Federal tem o único PPA que atinge a pontuação máxima nessa dimensão, uma vez que seus programas são os únicos que têm indicadores que medem resultados desagregados anualmente. Além disso, um total de 10 PPAs apresenta indicadores de resultados para o período de quatro anos, sendo que foram desconsiderados aqueles programas com indicadores exclusivamente de processo. Cabe ressaltar que 19 estados apresentam programas desagregados em atividades e projetos, 23 formularam corretamente os objetivos de cada programa e, por último, 24 alocaram cada um dos programas a alguma unidade responsável. Um total de 6 estados, Amazonas, Mato Grosso, Paraíba, Rio Grande do Sul, São Paulo e Tocantins, atinge uma pontuação de 0,2, o que significa que eles pontuaram positivamente em todas as dimensões, com exceção da dimensão referente à desagregação anual dos indicadores. Por outro lado, dois estados, Amapá e Mato Grosso do Sul, ficaram sem nenhuma pontuação nessa dimensão, mostrando que não foi desenvolvida a metodologia de elaboração de programas no planejamento estratégico.

Quadro 4 | D3. Dimensão programática $(0,25)$

\begin{tabular}{|l|l|l|l|l|l|l|}
\hline Estado & var7 & var8 & var9 & var10 & var11 & Pontos \\
\hline AC & Não & Não & Sim & Não & Sim & 0,1 \\
\hline AL & Sim & Sim & Não & Não & Sim & 0,15 \\
\hline
\end{tabular}




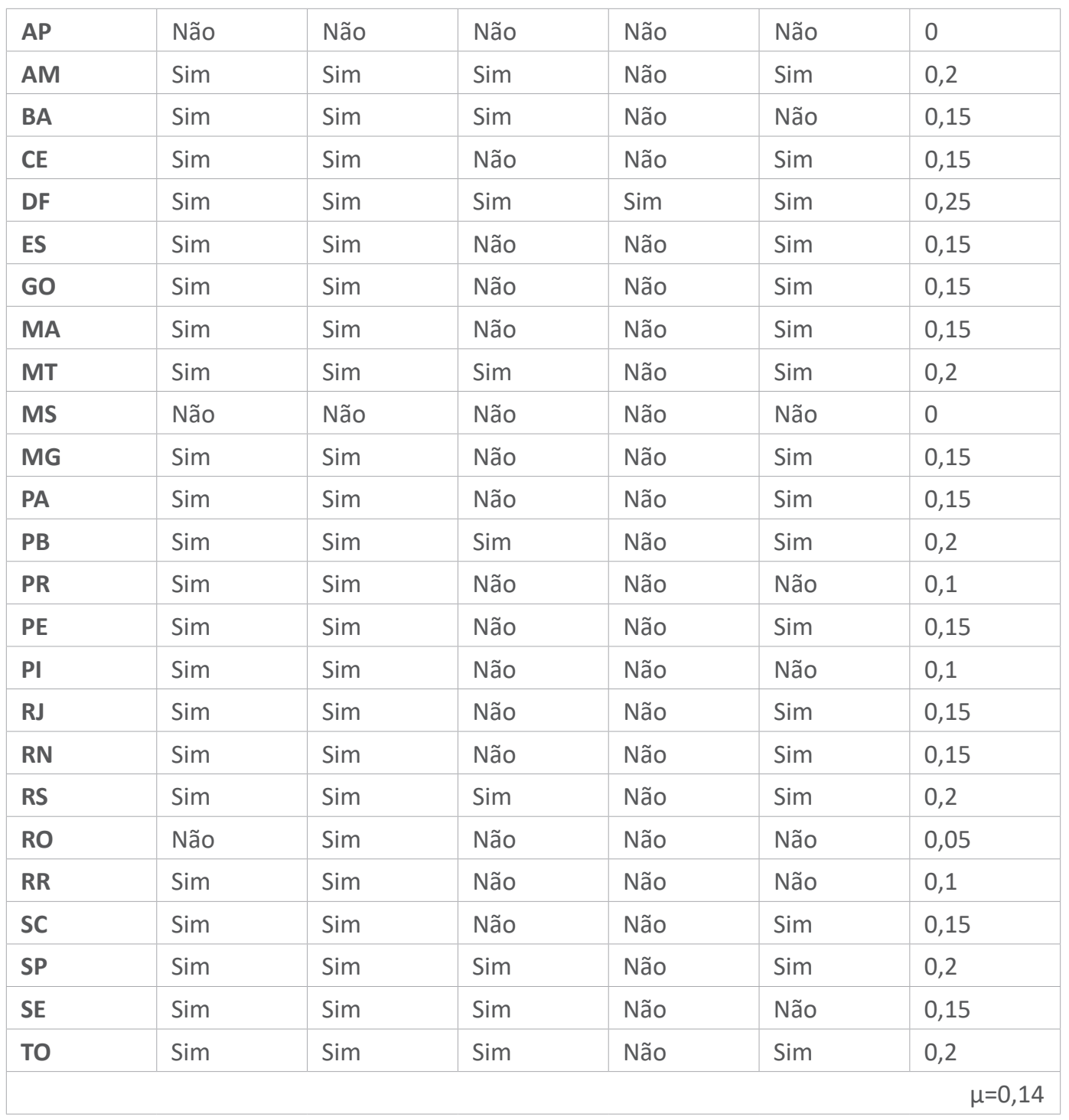

Fonte: elaboração própria.

Por último, a dimensão operacional mostra um resultado médio similar à dimensão anterior, de 0,14 pontos sobre 0,25, ou seja, um pouco acima da metade da maior pontuação possível. Quatro estados-Goiás, Maranhão, Minas Gerais e Rondônia - atingem a máxima pontuação, significando que todos os seus programas têm financiamento e que ele está desagregado anualmente e não simplesmente para o período de quatro anos. Além disso, os programas têm um código, que corresponde ao mesmo das sucessivas LOAs. Dois estados - Mato Grosso do Sul e Pará - têm pontuação 0 nessa dimensão, o 
que significa que seus programas não têm integração com a LDO e a LOA e, portanto, não têm capacidade de ser executáveis anualmente. Um total de 21 estados apresentam em seu PPA o código orçamentário, que tem seu correlato nos sucessivos orçamentos, o que mostra, em geral, uma correta integração entre as diferentes ferramentas de planejamento quatrienal e anual. Isso pode ser confirmado pelo fato de que 20 PPAs estaduais contam com financiamento detalhado para cada um dos programas. O grau de detalhamento desse financiamento quatrienal, no entanto, não atinge o nível anual, pois somente os 4 estados acima mencionados alcançaram a pontuação máxima nesse indicador, ou seja, são capazes de desagregar anualmente a estimativa de despesas para cada programa, ajustando assim de forma mais próxima PPA com LOA.

Quadro 5 | D4. Dimensão operacional $(0,25)$

\begin{tabular}{|l|c|c|c|c|}
\hline Estado & Var.12 & Var.13 & Var.14 & Pontos \\
\hline Acre & Sim & Não & Sim & 0,17 \\
\hline Alagoas & Sim & Não & Sim & 0,17 \\
\hline Amapá & Sim & Não & Sim & 0,17 \\
\hline Amazonas & Sim & Não & Não & 0,08 \\
\hline Bahia & Sim & Não & Não & 0,08 \\
\hline Ceará & Sim & Não & Sim & 0,17 \\
\hline Distrito Federal & Não & Não & Sim & 0,08 \\
\hline Espírito Santo & Sim & Não & Sim & 0,17 \\
\hline Goiás & Sim & Sim & Sim & 0,25 \\
\hline Maranhão & Sim & Sim & Sim & 0,25 \\
\hline Mato Grosso & Sim & Não & Sim & 0,17 \\
\hline M. Grosso do S. & Não & Não & Não & 0 \\
\hline Minas Gerais & Sim & Sim & Sim & 0,25 \\
\hline Pará & Não & Não & Não & 0 \\
\hline Paraíba & Sim & Não & Sim & 0,17 \\
\hline Paraná & Sim & Não & Sim & 0,17 \\
\hline Pernambuco & Sim & Não & Sim & 0,17 \\
\hline Piauí & Não & Não & Não & 0,17 \\
\hline Rio de Janeiro & Não & Não & Sim & 0,08 \\
\hline R.G. do Norte & Não & Não & Sim & 0,08 \\
\hline R.G. do Sul & Sim & Não & Não & 0,08 \\
\hline
\end{tabular}




\begin{tabular}{|l|c|c|c|c|}
\hline Rondônia & $\operatorname{Sim}$ & $\operatorname{Sim}$ & $\operatorname{Sim}$ & 0,25 \\
\hline Roraima & Não & Não & $\operatorname{Sim}$ & 0,08 \\
\hline Santa Catarina & $\operatorname{Sim}$ & Não & $\operatorname{Sim}$ & 0,17 \\
\hline São Paulo & $\operatorname{Sim}$ & Não & $\operatorname{Sim}$ & 0,17 \\
\hline Sergipe & $\operatorname{Sim}$ & Não & $\operatorname{Sim}$ & 0,17 \\
\hline Tocantins & $\operatorname{Sim}$ & Não & $\operatorname{Sim}$ & 0 \\
\hline & & & $\mu=0,14$ \\
\hline
\end{tabular}

Fonte: elaboração própria.

Uma vez consideradas conjuntamente as quatro dimensões, não é possível observar pautas geográficas ou relacionadas com o nível de PIB per capita, no melhor ou pior desempenho na elaboração dos PPAs. O que se observa de forma clara é o escasso nível de desempenho atingido em geral pelos estados na elaboração dos seus PPAs. A maioria dos estados, um total de 16 , concentra-se na faixa entre 0,2 e 0,5 pontos sobre um total de 1, o que indica um nível baixo. Além disso, temos o Estado de Roraima, que teve uma pontuação de 0,18 , considerada como muito baixa. Entre os estados que conseguiram um resultado positivo, somente Mato Grosso e Sergipe conseguiram uma pontuação tida como alta, entre 0,7 e 0,9 pontos, sendo que nenhum PPA estadual conseguiu ter um desempenho excelente, acima desse nível. Um total de 7 estados conseguiu uma pontuação considerada média, o que significa que, somando aos dois anteriormente citados, somente 9 , de 27 , conseguiram um desempenho acima da metade do indicador composto.

Quadro 6 | Total índice de qualidade dos PPAs estaduais segundo a GpR

\begin{tabular}{|l|c|c|c|c|c|c|}
\hline Estado & D1 & D2 & D3 & D4 & Pontos & Posição \\
\hline Acre & 0,08 & 0,08 & 0,1 & 0,17 & 0,43 & 15 \\
\hline Alagoas & 0,08 & 0,25 & 0,15 & 0,17 & 0,65 & 5 \\
\hline Amapá & 0,08 & 0,08 & 0 & 0,17 & 0,33 & 21 \\
\hline Amazonas & 0 & 0,17 & 0,2 & 0,08 & 0,45 & 12 \\
\hline Bahia & 0 & 0,08 & 0,15 & 0,08 & 0,31 & 24 \\
\hline Ceará & 0 & 0 & 0,15 & 0,17 & 0,32 & 23 \\
\hline Distrito F. & 0,08 & 0,25 & 0,25 & 0,08 & 0,66 & 3 \\
\hline Espírito Santo & 0,08 & 0,17 & 0,15 & 0,17 & 0,57 & 8 \\
\hline Goiás & 0 & 0,17 & 0,15 & 0,25 & 0,57 & 8 \\
\hline
\end{tabular}




\begin{tabular}{|l|c|c|c|c|c|c|}
\hline Maranhão & 0 & 0,08 & 0,15 & 0,25 & 0,48 & 10 \\
\hline Mato Grosso & 0,17 & 0,25 & 0,2 & 0,17 & 0,79 & 1 \\
\hline M. G. do Sul & 0,08 & 0,25 & 0 & 0,33 & 0,33 & 21 \\
\hline Minas Gerais & 0 & 0 & 0,15 & 0,25 & 0,4 & 16 \\
\hline Pará & 0,08 & 0,25 & 0,15 & 0 & 0,48 & 10 \\
\hline Paraíba & 0,08 & 0 & 0,2 & 0,17 & 0,45 & 12 \\
\hline Paraná & 0 & 0 & 0,1 & 0,17 & 0,27 & 26 \\
\hline Pernambuco & 0,17 & 0,17 & 0,15 & 0,17 & 0,66 & 3 \\
\hline Piauí & 0,08 & 0,17 & 0,1 & 0 & 0,35 & 20 \\
\hline Rio de Janeiro & 0 & 0,08 & 0,15 & 0,08 & 0,31 & 24 \\
\hline R.G. do Norte & 0,17 & 0,25 & 0,15 & 0,08 & 0,65 & 5 \\
\hline R.G. do Sul & 0,08 & 0,25 & 0,2 & 0,08 & 0,61 & 7 \\
\hline Rondônia & 0,08 & 0 & 0,05 & 0,25 & 0,38 & 17 \\
\hline Roraima & 0 & 0 & 0,1 & 0,08 & 0,18 & 27 \\
\hline S. Catarina & 0,08 & 0 & 0,15 & 0,17 & 0,4 & 16 \\
\hline São Paulo & 0 & 0,08 & 0,2 & 0,17 & 0,45 & 12 \\
\hline Sergipe & 0,17 & 0,25 & 0,15 & 0,17 & 0,74 & 2 \\
\hline Tocantins & 0 & 0 & 0,2 & 0,17 & 0,37 & 19 \\
\hline Fon & & & & & \\
\hline
\end{tabular}

Fonte: elaboração própria.

\section{Quadro 7 | Classificação}

\begin{tabular}{|c|}
\hline Muito alto $(\geq 0.9)$ \\
\hline- \\
\hline Alto $(<0.9 \geq 0.7)$ \\
\hline Mato Grosso, Sergipe \\
\hline Médio $(<0.7 \geq 0.5)$ \\
\hline $\begin{array}{c}\text { Distrito Federal, Pernambuco, Alagoas, Rio Grande do Norte, Rio Grande do Sul, Espírito Santo, } \\
\text { Goiás }\end{array}$ \\
\hline Baixo (0,5) \\
\hline Maranhão, Pará, Amazonas, São Paulo, Acre, Minas Gerais, Santa Catarina, Rondônia, \\
\hline Tocantins, Piauí, Amapá, Mato Grosso do Sul, Ceará, Bahia, Rio de Janeiro, Paraná \\
\hline Muito baixo \\
\hline Roraima \\
\hline
\end{tabular}

Fonte: elaboração própria. 
Sendo assim, a principal conclusão que se extrai deste estudo é que a qualidade do planejamento estratégico no nível estadual é baixa e que o caminho percorrido no momento de estabelecer o PPA como ferramenta de gestão ainda é insuficiente. A coalizão montada entre técnicos e acadêmicos envolvidos na questão do planejamento para transformar os PPAs estaduais em uma ferramenta que, para além de um documento burocrático, ajude na gestão pública, está longe de atingir um resultado satisfatório. Na elaboração do planejamento estratégico, as rotinas da administração pública procedimental ainda não foram substituídas de forma generalizada nos estados pelo planejamento orientado a resultados.

As dimensões que mais se aproximaram da elaboração orçamentária, ou seja, a dimensão programática e a dimensão operativa são as que atingiram maior pontuação. Contrariamente, a dimensão estratégica, ou seja, aquela que é precisamente a que deveria definir com clareza os grandes problemas e as metas a serem priorizadas, obteve uma pontuação bem abaixo do teto do índice. Em virtude disso, a segunda principal conclusão a ser extraída, além do insuficiente desenvolvimento do planejamento estratégico nos estados, é que os PPAs de forma geral ainda continuam sendo concebidos como orçamentos para quatro anos. Portanto, os estados que mais têm desenvolvido a técnica do orçamento no programa são os que mais têm desenvolvido os seus PPAs. No entanto, em geral, esses planos ainda estão longe de se converter em documentos operativos, que consigam priorizar uma série de linhas estratégicas, por meio de um diagnóstico, de converter tais linhas estratégicas em objetivos estruturadores e, a partir dessa técnica, elaborar programas que possam ter o seu reflexo no orçamento anual, sendo acompanhados mediante indicadores de desempenho. 
Figura 1 | Índice de qualidade dos PPAs estaduais

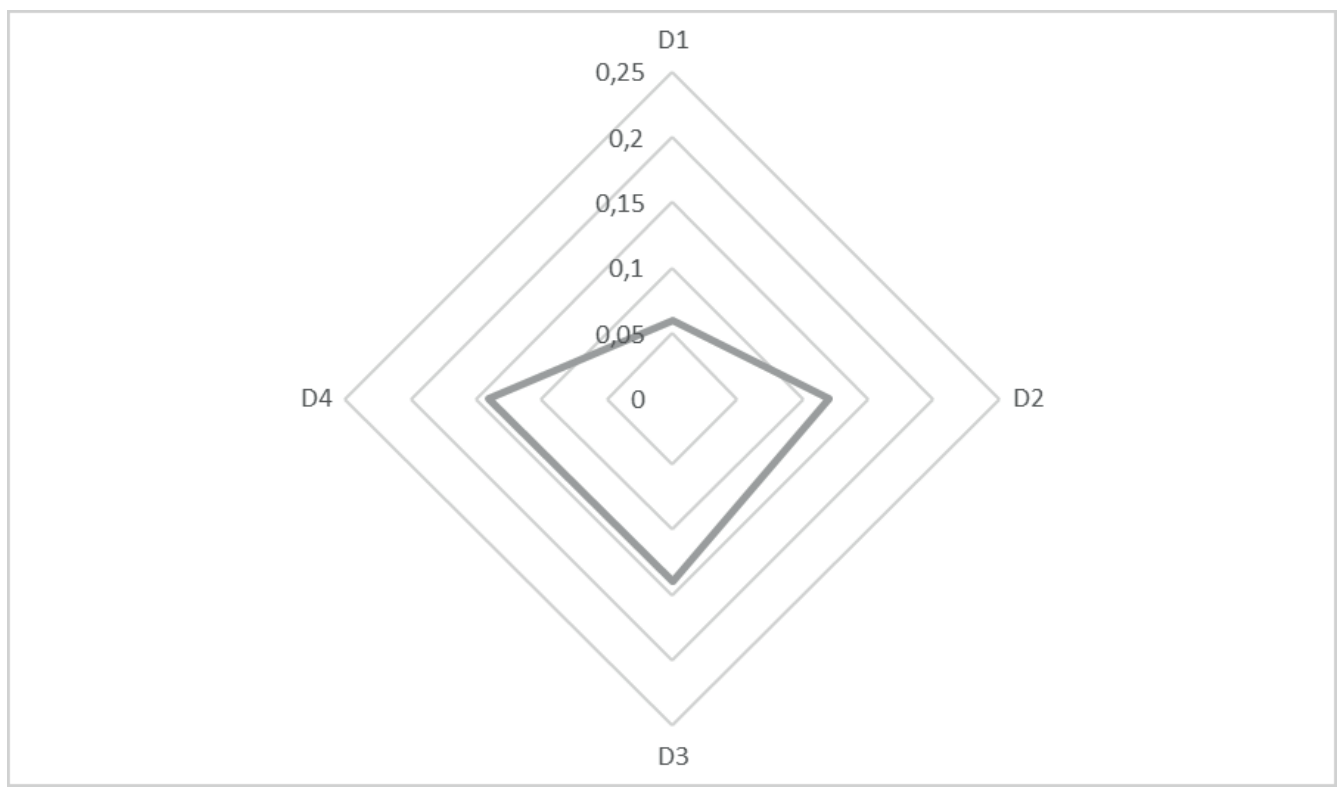

Fonte: elaboração própria.

\section{Conclusão}

Este artigo analisou a implantação do planejamento estratégico nos diferentes estados brasileiros, por intermédio de um diagnóstico da penetração desse paradigma de administração pública nos distintos planos plurianuais estaduais. Dado que o planejamento é a primeira das fases do modelo de gestão focado no desempenho, foi entendido que avaliar os diferentes PPAs dos estados, durante o período de 2016 a 2019, fornece uma imagem ajustada do seu grau de avanço nos níveis subnacionais de governo. Para isso, após revisar a literatura sobre o planejamento estratégico focado no desempenho na administração pública, desenvolveu-se um índice, composto por quatro dimensões, para avaliar a qualidade dos PPAs aqui considerados.

Os resultados apresentados mostram um insuficiente desenvolvimento da técnica de planejamento estratégico orientado à gestão pública. Como foi mostrado, isso se deu em razão de a maioria dos PPAs ser concebida como orçamentos para um período de 
quatro anos, e não como uma ferramenta capaz de diagnosticar os desafios externos e as capacidades internas e, a partir de então, estabelecer linhas estratégicas. Além disso, foi detectado um insuficiente desenvolvimento do uso de indicadores para medir resultados, principalmente indicadores de acompanhamento anual.

Este estudo buscou apresentar a situação do planejamento estratégico, na sua relação com as técnicas da administração pública orientadas à obtenção de objetivos no longo prazo, num determinado momento do tempo que corresponde aos PPAs estaduais do período 2016-2019. Porém, estima-se que não foram esgotadas nem as possibilidades metodológicas da sua aplicabilidade para outros níveis de governo, nem a capacidade para fazer estudos longitudinais que possam ajudar a analisar a evolução do fenômeno ao longo do tempo. Logo, é desejável que este artigo possa ajudar na consolidação de uma agenda de pesquisa, ainda incipiente, que continue avaliando a expansão de técnicas de gestão pública orientada ao desempenho e à melhora do planejamento estratégico no setor público.

\section{Referências bibliográficas}

Abrucio, F; SegatTo, C. I. A gestão por resultados na educação em quatro estados brasileiros. Revista do Serviço Público, v. 68, p. 85-106, 2017.

ABRUCIO, F; GAETANI, F. Avanços e perspectivas da gestão pública nos estados: agenda, aprendizado e coalizão. In: CosTA, L. L. Avanços e perspectivas da gestão pública nos Estados. Rio de Janeiro: Qualitimark, 2008.

ALMEIDA, P. R. A experiência brasileira em planejamento econômico: uma síntese histórica. In: GIACOMINI, J; PAGNUSSAT, J. L. Planejamento e orçamento governamental: volume 1. Brasília: Enap, 2006.

BRYSON, J. Strategic planning for public and nonprofit organizations. San Francisco: John Wiley. 2004

Centro latinoamericano de Administración para el Desarrollo (Clad). Modelo abierto de gestión para resultados en el sector público. Revista del CLAD Reforma y Democracia, v. 39, p. 149-210, 2007.

Centro latinoamericano de Administración para el Desarrollo (Clad). Modelo abierto de Gestión para Resultados en el sector público. Washington D.C: BID, 2011. 
CARDoso, J. C. A reinvenção do planejamento governamental no Brasil: diálogos para o desenvolvimento. Brasília: Ipea, 2011.

CARNEIRO, R. PPAs estaduais em perspectiva comparada: processos, conteúdo e monitoramento. In: CARDOSO, J. C; SANTOS, E. V. PPA 2012-2015: experimentalismo institucional e resistência burocrática. Brasília: Ipea, 2015.

Couto, L. F. Planos Plurianuais e estratégias de desenvolvimento. In: GIMENE, M; CoUTO, L. F. Planejamento e orçamento público no Brasil. Brasília: Enap, 2017.

CUNILL, N; OSPINA, S. Performance measurement and evaluation systems: institucionalizing accountability for governmental results in Latin America. New directions for evaluation, v. 134, p. 77-91, 2012.

CHICA VÉLEZ, S. Gestión para resultados en el desarrollo: hacia la construcción de buena gobernanza. Administración y Desarrollo, v. 45, n. 1, p. 71-93, 2015.

DE TONI, J. O planejamento estratégico governamental: reflexões metodológicas e implicações na gestão pública. Curitiba: Intersaberes, 2016.

DE TONI, J. A retomada do planejamento estratégico governamental no Brasil: novos dilemas e perspectivas. In: GIMENE; CoUto, L. F. Planejamento e orçamento público no Brasil. Brasília: Enap, 2017.

DRUCKER, P. The practice of management. New York: Harper and Row, 1954.

DRUCKER, P. Managing for results: economic tasks and risk taking decisions. New York: Harper and Row, 1964.

DUSSAGE, M. Los orígenes de la gestión por resultados en Chile y México: ¿imposición, copia o aprendizaje transnacional? Revista mexicana de análisis político y administración pública, v. 4, n. 2, p. 89-110, 2015.

DUSSAGE, M. Introducción a la gestión para resultados. In Dussage, M. (ed.). Gestión para resultados. México D.F: Siglo XXI, 2016.

GarcÉs, A; SIlveIRA, J. P. Gestão pública orientada para resultados no Brasil. Revista do Serviço Público, v. 53, n. 4, p. 53-77, 2002.

GARCIA, R. C. A reorganização do processo de planejamento do governo federal: o PPA 2000-2003. In: CARdoso, J. C; CUnhA, A. S. Planejamento e avaliação de políticas públicas. Brasília: Ipea, 2015a.

GARCIA, R. C. PPA: o que não é e o que pode ser. In: CARDoso, J. C; CunhA, A. S. Planejamento e avaliação de políticas públicas. Brasília: Ipea, 2015b.

GARCÍA LóPEZ, R; GARCÍA MORENO, M. Managing for development results: progress and challenges in Latin America and the Caribbean. Washington, BID, 2010.

KaUfmann, J., SAnginés, M; García Moreno, M. Construyendo gobiernos efectivos: logros y retos de la gestión pública para resultados en América Latina y el Caribe. Washington: BID, 2015. 
MILANESI, A. La gestión por resultados: una revisión de la literatura y sus desafíos en América Latina. Administración y Desarrollo, v. 48, n. 1, p. 170-199, 2018.

NATHAN, R. Introduction. In: FORSHITE, D. W. Quicker, better, cheaper?: managing performance in American government. New York: The Rockefeller Institute Press, 2001.

PARES, A; VAlle, B. A retomada do planejamento governamental no Brasil e seus desafios. In: GIACOMONI, J; PAGNUSSAT, J. L. Planejamento e orçamento governamental: volume 1. Brasília: Enap, 2006.

PolLIT, C. Performance management 40 years on: a review. Some key decisions and consequences. Public money \& management, v. 38, n. 3, p. 167-174, 2018.

POLLIT, C; BOUCKAERT, G. Public management reform: a comparative analysis -New Public Management, Governance and the Neo-Weberian State. Oxford: Oxford University Press, 2001.

REZENDE, F. Planejamento no Brasil: auge, declínio e caminhos para a reconstrução. In:

TAlBot, C. Performance management. In: Ferlye, E.; LYNN, L; Pollit, C. The Oxford hadbook of public management. Oxford: Oxford University Press, 2007.

WALTERS, J.; ABRAHAMS, M; FountAIN, J. Managing for results: an overview. Washington D.C: Governmental Accounting and Standards Board, 2003.

\section{Juan Vicente Bachiller}

(D) https://orcid.org/0000-0002-0639-2434

Doutor em Ciência Política pela Universidade de Salamanca (USAL), com Pós-Doutorado no Instituto de Estudos Sociais e Políticos da Universidade do Estado do Rio de Janeiro (IESP-UERJ). É professor de Políticas Públicas na Universidade Federal Fluminense (UFF).

E-mail: bachiller_juan@id.uff.br 